\title{
Effectiveness of Adjunctive Corticosteroid Therapy in Patients with Severe COVID-19: A Retrospective Cohort Study
}

\section{Bin Xiong}

The Second Affiliated Hospital of Chongqing Medical University

\section{Yuan-Yuan Qin}

Chongqing Public Health Medical Center

\section{Li-Min $\mathrm{He}$}

The First Branch of The First Affiliated Hospital, Chongqing Medical University

Hu Du

The Second Affiliated Hospital of Chongqing Medical University

\section{Zhu Zhan}

The Second Affiliated Hospital of Chongqing Medical University

\section{Yi-Hong Zhou}

Chongqing Public Health Medical Center

\section{Yao-Kai Chen}

Chongqing Public Health Medical Center

\section{An Zhang ( $\nabla$ 300704@hospital.cqmu.edu.cn )}

The Second Affiliated Hospital of Chongqing Medical University

\section{Research Article}

Keywords: COVID-19, Corticosteroid, SARS-COV-2, Outcomes

Posted Date: October 8th, 2020

DOI: https://doi.org/10.21203/rs.3.rs-89097/v1

License: (a) (i) This work is licensed under a Creative Commons Attribution 4.0 International License. Read Full License 


\section{Abstract}

Objective: To investigate the effectiveness of adjunctive corticosteroid therapy in patients with severe COVID19.

Methods: We conducted a retrospective analysis of the difference in several outcomes between patients with severe COVID-19 who received corticosteroid therapy (the corticosteroid group) and patients with severe COVID-19 who did not receive corticosteroid therapy (the non-corticosteroid group).

Results: Seventy-five patients were included in this study. Of these, 47 patients were in the corticosteroid group and 28 patients were in the non-corticosteroid group. There were no differences between the two groups in the total length of hospital stay (LOS), the length of ICU stay, high-flow oxygen days, non-invasive ventilator days, invasive ventilation days, and mortality rate. Total lesion volume ratio, consolidation volume ratio and GGO volume ratio in the corticosteroid group decreased significantly on day 14 , while those in the non-corticosteroid group did not show a significant decrease.

Conclusions: Our results show that adjunctive corticosteroid use did not significantly improve clinical outcomes in severe COVID-19 patients, but might promote the absorption of pulmonary lesions. Larger multicenter randomized controlled studies may be needed to confirm this.

\section{Introduction}

The outbreak of coronavirus disease 2019 (COVID-19) is currently a serious global public health challenge caused by a novel coronavirus, severe acute respiratory syndrome coronavirus 2 (SARS-CoV-2), which is an enveloped ribonucleic acid (RNA) virus and is mainly transmitted through the respiratory tract, causing damage to the human respiratory system, systemic inflammatory reactions, and multiple organ failure in certain cases $[1,2]$. Prior to the COVID-19 outbreak, two similar coronavirus outbreaks had already occurred in Asia, including the outbreak of SARS-CoV in 2003 [3] and the outbreak of Middle East respiratory syndrome coronavirus (MERS-CoV) in 2012 [4, 5].

According to the existing literature, the symptoms of patients with COVID-19 are non-specific, and include fever, cough and myalgia, accompanied by diarrhea, with or without the subsequent development of dyspnea. Severe cases involve acute respiratory distress syndrome (ARDS), sepsis, and septic shock [6-10]. While symptomatic supportive treatment may be administered, there is currently no specific antiviral treatment for COVID-19. Different organizations, authoritative institutions, and scholars have successively released a variety of COVID-19 diagnosis or treatment plans or opinions, but drug treatment recommendations have been inconsistent. Corticosteroids have an inhibitory effect on inflammatory factors, and are often used as an adjuvant treatment for viral pneumonia. Studies have shown that the use of corticosteroids can help suppress excessive inflammatory reactions; nevertheless, the use of corticosteroids may delay the elimination of the virus and may result in serious adverse effects [11]. So far, the effectiveness of systemic corticosteroid use in patients with COVID-19 remains unknown. The aim of the present study was to investigate the effectiveness of adjunctive corticosteroid administration in patients with severe COVID-19. 


\section{Materials And Methods}

\section{Ethics, consent and permissions}

The Ethics Commission of the Chongqing Public Health Medical Center approved this study (No.2020-025$\mathrm{KY}$ ). Written informed consent was waived due to the rapid emergence of this infectious disease.

\section{Study design and patients}

The present study is a retrospective cohort study, which included all severe COVID-19 patients admitted to Chongqing, Chongqing Public Health Medical Center and Chongqing Three Gorges Central Hospital from January to March, 2020.

The diagnosis of severe COVID-19 in subjects had to meet the following criteria: (1) Identification of COVID19 via real-time reverse transcription-polymerase chain reaction (RT-PCR) and/or next-generation sequencing (NGS); (2) Having at least one of the following conditions $₫ a$. Respiratory distress ( $\geq 30$ times/min); $b$. Oxygen saturation $\leq 93 \%$ at rest; $\mathrm{c}$. Oxygenation index $\left(\mathrm{PaO}_{2} / \mathrm{FiO}_{2}\right) \leq 300 \mathrm{mmHg}$; d. Respiratory failure requiring mechanical ventilation; e. Septic shock development; $f$. Critical organ failure requiring intensive care unit (ICU) care. Shock was defined according to the World Health Organization (WHO) guidelines for COVID-19 [12]. Acute kidney injury was identified on the basis of serum creatinine [13]. Cardiac injury was diagnosed if the serum concentration of hypersensitive cardiac troponin I (hsTNI) was above the upper limit of the reference range ( $>28 \mathrm{pg} / \mathrm{mL})$, measured in the laboratory of the designated hospital.

All patients received similar conventional COVID-19 treatment according to the Chinese Clinical Guidance for COVID-19 Pneumonia Diagnosis and Treatment (Trial Version 5) [14]. We stratified the included patients into two groups according to whether they were administered corticosteroids in their treatment regimens or not, namely, the corticosteroid group and the non-corticosteroid group.

\section{Data collection}

We reviewed clinical electronic medical records, nursing records, laboratory findings, and radiological examinations for all patients with the diagnosis of severe COVID-19. The admission data of these patients from day 0 (D 0), which was defined as the day of severe COVID-19 diagnosis to discharge or death were collected. Data were evaluated and collated, by using case record forms. We collected data of gender, age, smoking, vital signs (heart rate, respiratory rate, mean arterial pressure), chronic medical history (hypertension, diabetes, coronary heart disease (CAD), chronic obstructive pulmonary disease (COPD), cerebrovascular disease, viral hepatitis, neoplastic disease, chronic kidney disease (CKD), symptoms from onset to hospital admission (fever, cough, sputum, dyspnea, weakness, headache, diarrhea), treatment (oxygen therapy, antibiotic agents, antifungal agents, immunoglobulin, thymopentin (or thymalfasin), and extracorporeal membrane oxygenation (ECMO), acute physiology and chronic health assessment (APACHE) II score, as well as total length of hospital stay (LOS), and length of ICU stay.

Routine blood test results were also collected, including blood gas analysis, complete blood count, and serum biochemical tests, including electrolytes, lactate dehydrogenase, liver and kidney function, 
coagulation function, cardiac enzymes, interleukin-6 (IL-6), cluster of differentiation (CD)-4-positive T-cell count, CD-8-positive T-cell count, C-reactive protein (CRP), D-dimer, and procalcitonin (PCT). Chest radiographs or computerized tomography (CT) scans were also performed for all hospitalized patients, and the frequency of examinations was determined by the attending physician.

\section{Outcome assessment}

We assessed the changes in APACHE II score and oxygenation index $\left(\mathrm{PaO}_{2} / \mathrm{FiO}_{2}\right)$, the changes in lymphocyte count, CD4+ T-cell count, CD8+ T-cell count, pulmonary lesion volume, LOS, and length of ICU stay, proportions of patients using mechanical ventilation, and mortality rate.

The measurement of the extent of pneumonia was performed with the FACT Medical Imaging System (Dexin Medical Imaging Technology Co, Ltd.), in which Pulmonary Infection Assisted Diagnosis (V1.7.0.1) automatically segmented the entire lung and lesions depicted on CT images using the "break and repair" shape analysis strategy, and then calculated the volumes of the entire lung and all lesions. Based on the initial automatic segmentation, the boundaries of each lesion were further precisely adjusted by manual tools to avoid the influence of non-inflammatory lesions such as large blood vessels, pleural effusion, pleural thickening, and calcification. In addition, according to the definitions of ground-glass opacity (GGO) and consolidation in the Fleischner Society Recommendations $[15,16]$, we further measured the volume occupied by GGOs and consolidation components in the lesions. After completing all adjustments, the system automatically calculated the volumes of the lesions, GGOs, consolidation, and that of the whole lung. Finally, the volume ratio was calculated by the following formulas: total lesion volume ratio = (volume of all lesions)/(volume of whole lung) $\times 100 ; \mathrm{GGO}$ volume ratio $=$ (volume of GGOs)/(volume of whole lung) $\times 100$; and consolidation volume ratio $=($ volume of consolidation $) /($ volume of whole lung $) \times 100$.

\section{Statistical Analysis}

The data were analyzed with IBM SPSS software version 26 (IBM-SPSS Inc., Chicago, IL, USA). The corticosteroid group and the non-corticosteroid group were initially analyzed by descriptive statistical methods. We performed the Kolmogorov-Smirnov test to evaluate the normality of the data distributions. Continuous variables are presented as the mean $\pm S D$, and independent samples were analyzed by the t-test. Categorical variables were analyzed using the Chi-squared test or the Fisher's exact test. Differences were statistically significant at $p<0.05$.

\section{Results}

\section{Demographics and baseline characteristics}

The demographic and clinical characteristics of the patients are summarized in Table 1. Seventy-five patients with severe COVID-19 were included in this study. The mean age was $58.92 \pm 14.29$ years, and 39 (52\%) patients were male. Of the 75 patients, 47 (62.7\%) were administered corticosteroids and were therefore classified into the corticosteroid group, whereas 28 (37.3\%) were not administered corticosteroids and were therefore classified into the non-corticosteroid group. All the 47 patients in the corticosteroid group 
received corticosteroids use within 72 hours of severe COVID-19 diagnosis. The symptoms of COVID-19 in the included patients were fever (61.33\%), cough (76\%), dyspnea (53.33\%), sputum production $(34.67 \%)$, and weakness $(29.33 \%)$. There were no statistically calculated differences between the two groups of patients in terms of gender, age, smoking, APACHE II score, hypertension, diabetes, CAD, COPD, cerebrovascular disease, viral hepatitis, neoplastic disease, CKD, fever, cough, sputum, weakness, headache, diarrhea, shock, leukopenia, thrombocytopenia, liver dysfunction, AKl, or cardiac injury, as displayed in Table 1.

\section{Treatments and outcomes in patients with severe COVID-19}

Approximately eighty-three percent of the patients (62/75) received antibiotic therapy, and $12(16 \%)$ of the patients received antifungal therapy. Sixty three patients $(84 \%)$ received thymopentin or thymalfasin, and 30 patients $(42.76 \%)$ received intravenous injection (IV) immunoglobulin. 28 patients (37.33\%) received noninvasive ventilation (NIV), 7 patients $(9.33 \%)$ received invasive ventilation, and three patients $(4 \%)$ received ECMO therapy.

In the corticosteroid group, the vast majority of enrolled patients (95.74\%) received antibiotics, and $25.53 \%$ of the patients received antifungal therapy; the proportion of those patients receiving antibiotics and antifungals was significantly higher in the corticosteroid group compared to the non-corticosteroid group ( $p$ $<0.05$ ). Nevertheless, we found no differences between the two groups in the total LOS, the length of ICU stay, the high-flow oxygen days, the NIV days, the invasive ventilator days, and the mortality rate, as indicated in Table 2.

\section{Comparison of laboratory variables}

There were no significant differences in APACHE II scores between the corticosteroid group and the noncorticosteroid group on D 0 or D14. Moreover, the APACHE II score significantly decreased in each of the two groups from D 0 to D14 $(p<0.05)$, as shown in Table 3.

We did not observe a significant difference in the oxygenation index between the corticosteroid group and the non-corticosteroid group on D 0 or D14. In addition, the oxygenation index in each of the two groups significantly increased from D 0 to D14 $(p<0.05)$.

At the same time, we found that inflammatory indicators at baseline (e.g., CRP, PCT, WBC, D-dimer, IL-6, and IL-10) and some serum biochemical indicators were no significant differences in those indicators between the two groups on D 0 or D14.

Some inflammatory factors in corticosteroid group, including D-dimer, IL-10, increased over time, while CRP decreased significantly from D 0 to D14 $(p<0.05)$. And some inflammatory factors in the non-corticosteroid group, including PCT, CRP, IL-10, decreased significantly from D 0 to D14 $(p<0.05)$.

Subsequently, compared with the non-corticosteroid group, we found that CD4+, but not CD8+ T-cell counts were significantly decreased in both groups from D 0 to D14 $(p<0.05)$. This is illustrated in detail in Table 3.

\section{The results of imaging evaluation in patients with COVID-19}


Table 4 shows the results of imaging evaluations in each group of patients with severe COVID-19. We found that the common chest CT features of COVID-19 patients mainly included consolidation, GGOs, and linear opacities. The total lesion volume ratio, consolidation volume ratio, and GGO volume ratio were significantly higher in the corticosteroid group than in the non-corticosteroid group on D $0(p<0.05)$. However, there was no significant difference in these indicators between the two groups on D14 $(p>0.05)$. This is illustrated in Fig 1.

We found that total lesion volume ratio and GGO volume ratio in both groups decreased over time, while total lesion volume ratio and GGO volume ratio in the corticosteroid group decreased significantly on D14, which is different with that in the non-corticosteroid group. However, unlike patients in the corticosteroid group, whose consolidation volume ratio decreased significantly on D14, we found that the consolidation volume ratio in the non-corticosteroid group increased significantly on D14.

\section{Discussion}

In this study, we found that the levels of inflammatory indicators (e.g., CRP, PCT, WBC, D-dimer, IL-6, and IL10) were increased in patients with COVID-19, counts of lymphocytes, CD4+, and CD8+ T-cells were decreased in patients with COVID-19, and the common chest CT features in COVID-19 patients were consolidation and GGOs, compared with the normal reference value. The lymphocyte, CD4+, and CD8+ T-cell counts were significantly reduced in the adjunctive methylprednisolone corticosteroid group compared with those in the conventional corticosteroid group, but there were no significant differences in the APACHE II scores, oxygenation indices $\left(\mathrm{PaO}_{2} / \mathrm{FiO}_{2}\right)$, and LOS between these groups. The proportion of patients receiving mechanical ventilation was significantly higher in the corticosteroid group than in the noncorticosteroid group. Among the other parameters, only the duration of NIV was significantly different between the groups. In addition, 4 patients died in the corticosteroid group and no patients died in the noncorticosteroid group.

It is well known that in the treatment of viral pneumonia, the intermediate-acting corticosteroid methylprednisolone sodium succinate is commonly used because of its rapid onset, short biological half-life, good safety profile, and weak inhibition of the hypothalamus-pituitary-adrenal (HPA) axis; in addition, it is the only steroidal drug available for shock therapy [11]. In the treatment of COVID-19 patients, clinicians use corticosteroids mainly because of its anti-inflammatory effects [17]. Corticosteroids can act on various stages and suppress multiple links of the immune response. In the early stages of inflammation, corticosteroids can reduce capillary dilation, inflammatory cell exudation, leukocyte infiltration, and phagocytosis. In later phases of the inflammatory response, corticosteroids can inhibit the excessive proliferation of capillaries and fibroblasts [18]. Corticosteroids can act on corticosteroid receptors, inhibit nuclear transcription factor nuclear factor-kappa B (NF-kB) signaling, and further inhibit the transcription and translation of inflammatory factors. They may also play a role by inhibiting IL-2 gene transcription and then inhibiting T-cell clonal proliferation. Furthermore, corticosteroids can inhibit the induction of the immune

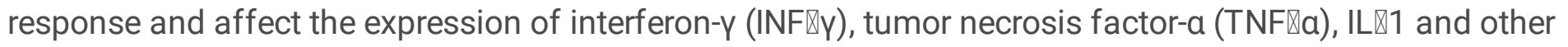
cytokines [19]. 
Pathological reports of patients who died from COVID-19 indicate that the lungs displayed alveolar injury with fibro-mucosal exudation, alveolar epithelial detachment, and pulmonary hyaline membrane formation, consistent with ARDS; inflammatory infiltration of mononuclear cells, mainly lymphocytes, in the interstitium was seen in the lungs, and the overall pathological changes were similar to that observed in patients who died from SARS and MERS [20]. Pathological findings of patients with SARS showed significant increases in $T$ and $B$ lymphocyte apoptosis, and mononuclear and macrophage numbers in immune organs. Similarly, in patients who died of COVID-19, there was a decrease in peripheral blood lymphocytes, but lymphocytes were also over-activated, while the numbers of highly pro-inflammatory CCR4+, CCR6+, and Th17 cells increased. The above results suggest that there is a severe reduction in anti-inflammatory immune cells, and an increase in immune response-related damage in the lungs [20]. Similarly, the results of previous studies showed that $44.4 \%(12 / 27)$ of patients with mild COVID-19 and $84.6 \%(11 / 13)$ of patients with severe COVID-19 had lymphopenia. The absolute count of peripheral blood lymphocytes in patients with severe COVID-19 was significantly lower than that in patients with mild COVID-19; the counts of CD3+, CD4+, and CD8+ T-cell subsets were lower in the patients with severe COVID-19 than in the patients with mild COVID-19 [21]. Recent studies have shown that the numbers of total T-cells, CD4+ T-cells, and CD8+ T-cells were significantly reduced in COVID-19 patients [22], which is consistent with our findings. We also found that compared with the patients in the conventional corticosteroid group, COVID19 patients treated with adjunctive methylprednisolone had significantly lower absolute counts of CD4+ and CD8+ T-cells. This is similar to previous reports by Ghoneim et al., who found that the early use of corticosteroids (dexamethasone) to treat flu in rats significantly reduced the counts of CD4+ and CD8+ T-cells on day 7 [23]. The possible reason for this is that the use of corticosteroids may cause systemic immunosuppression [24, 25], which has a cytotoxic effect on CD4+ T-cells [26] and is not conducive to viral elimination, resulting in delayed virus clearance and accelerated disease progression.

In the early course of corticosteroid treatment of viral pneumonia, studies have shown that the adverse effects of glucocorticoid use are significantly greater than the anti-inflammatory effects. In patients with SARS infections, long-term high-dose corticosteroids were used, and there were serious adverse reactions such as invasive fungal infections and femoral head necrosis [27]. Brun-Buisson et al [28] studied the use of corticosteroids for the treatment of ARDS caused by H1N1 virus and found that the incidence of acquired pneumonia and death was higher in patients with the early use of corticosteroids than in those who did not receive corticosteroid therapy. In addition, the duration of mechanical ventilation in severely ill patients using corticosteroids was significantly prolonged [28]. In reports of adverse reactions in patients with SARS treated with corticosteroids, the adverse reactions were often positively correlated with the dose and timing of administration of corticosteroids [29]. Earlier use of corticosteroids to treat mild SARS and MERS patients was found to increase SARS viral load in patients, and to delay the clearance of MERS virus RNA [30, 31]. A meta-analysis published in 2019 that included ten observational studies on influenza found that the mortality rate, LOS in the ICU, and incidence of secondary bacterial or fungal infections were increased in patients receiving corticosteroids [32]. However, it is worth noting that a series of clinical trials have shown that when low-dose or physiological-dose corticosteroids are used to treat patients with septic shock caused by lung infection, the use of corticosteroids can significantly reduce the mortality of such patients, and can reverse shock, shorten the ICU LOS, and reduce the use of mechanical ventilation [33,34]. However, our study 
found that adjunctive corticosteroids did not significantly improve the oxygenation index $\left(\mathrm{PaO}_{2} / \mathrm{FiO}_{2}\right.$ ratio), shorten the LOS (including ICU stay), reduce mechanical ventilation usage time, or decrease mortality. At the same time, Wang et al. [35] found that no positive effects on outcomes were observed after the use of methylprednisolone. However, recent studies have found that systemic corticosteroid treatment in the initial 3 to 5 days can enhance the ratio of blood oxygen saturation and arterial oxygen tension to the oxygen fraction in patients with severe COVID\&19, and ventilation can further enhance these two indicators [36]. Unlike COVID $₫ 19$ patients with ARDS, COVID $₫ 19$ patients with shock or multiple organ injury derive no additional survival benefit from corticosteroid therapy. However, the use of corticosteroids can significantly inhibit the inflammatory cytokine storm that occurs in the ARDS stage in COVID-19 patients, giving patients valuable time to control the infection, and preventing secondary multiple organ damage and septic shock [36].

It is well known that corticosteroids do not directly inhibit viral replication. Their main role is to reduce inflammation and suppress the immune response. The anti-inflammatory effect is mainly manifested in the reduction of alveolar exudation and capillary permeability. The use of corticosteroids can delay the progression of mild pneumonia to ARDS, which can alleviate the occurrence of pulmonary fibrosis [37]. Studies have shown that when SARS patients have increased shadows on lung imaging and increased dyspnea, the early and appropriate use of corticosteroids can significantly improve the patients' clinical symptoms, reduce the degree of disease progression, and accelerate the absorption of lung lesions [38, 39]. Our study found that the addition of methylprednisolone can significantly improve lung lesions in patients with severe COVID-19 on D14. We found that clinicians prefer to use corticosteroids in patients with more extensive lung lesions. This may be related to the fact that pulmonary lesions were significantly worse in the corticosteroid group than in the non-corticosteroid group at baseline. These patients were critically ill, progressed rapidly, were difficult to treat, and required enhanced immunotherapy.

To a certain extent, our findings have several biases, which are inherent flaws of retrospective studies. In addition, due to the low numbers of patients with severe COVID-19 in Chongqing $₫ a$ limited number of critically ill patients were included in this study. Studies utilizing a larger sample size are warranted.

\section{Conclusion}

In summary, we found that adjunctive corticosteroids do not significantly improve clinical outcomes in patients with severe COVID-19. Larger multicenter randomized controlled studies may be needed to confirm this.

\section{Declarations}

\section{Author Contributions}

Conceived and designed the experiments: An Zhang,Yao-Kai Chen. Collected the epidemiological and clinical data: Hu Du, Zhu Zhan and Bin Xiong. Analyzed the data: Bin Xiong and Li-Min He. Contributed to the writing of the manuscript: Bin Xiong and Li-Min He. Revised the final manuscript: Yuan-Yuan Qin, Yi-Hong Zhou, Yao-Kai Chen And An Zhang. The authors read and approved the final manuscript. 
Acknowledgements

We thank all the patients involved in the study. The Ethics Commission of the Chongqing Public Health Medical Center approved this study (No.2020-025-KY).

\section{Funding}

This work was supported by the Chongqing Special Research Project for Novel Coronavirus Pneumonia Prevention and Control (No. cstc2020jscx-fyzxX0012; cstc2020jscx-fyzx0074) and Emergency Research Project of COVID-19 of Chongqing Health Commission (No. 2020NCPZX04).

\section{Conflict of interest}

The authors declared that they have no conflicts of interest to this work.

\section{References}

1. Gao GF. From "A"IV to "Z"IKV: Attacks from Emerging and Re-emerging Pathogens. Cell 2018; 172:11579.

2. Heymann DL, N Shindo. COVID-19: what is next for public health? Lancet (London, England) 2020; 395:542-5.

3. Zhong NS, BJ Zheng, YM Li, et al. Epidemiology and cause of severe acute respiratory syndrome (SARS) in Guangdong, People's Republic of China, in February, 2003. Lancet (London, England) 2003; 362:13538.

4. Zaki AM, S van Boheemen, TM Bestebroer, AD Osterhaus, RA Fouchier. Isolation of a novel coronavirus from a man with pneumonia in Saudi Arabia. The New England journal of medicine 2012; 367:1814-20.

5. Liu J, X Zheng, Q Tong, et al. Overlapping and discrete aspects of the pathology and pathogenesis of the emerging human pathogenic coronaviruses SARS-CoV, MERS-CoV, and 2019-nCoV. Journal of medical virology 2020; 92:491-4.

6. Rodriguez-Morales AJ, JA Cardona-Ospina, E Gutiérrez-Ocampo, et al. Clinical, laboratory and imaging features of COVID-19: A systematic review and meta-analysis. Travel medicine and infectious disease 2020:101623.

7. Huang C, Y Wang, X Li, et al. Clinical features of patients infected with 2019 novel coronavirus in Wuhan, China. Lancet (London, England) 2020; 395:497-506.

8. Wang C, PW Horby, FG Hayden, GF Gao. A novel coronavirus outbreak of global health concern. Lancet (London, England) 2020; 395:470-3.

9. Chan JF, S Yuan, KH Kok, et al. A familial cluster of pneumonia associated with the 2019 novel coronavirus indicating person-to-person transmission: a study of a family cluster. Lancet (London, England) 2020; 395:514-23.

10. Chen N, M Zhou, X Dong, et al. Epidemiological and clinical characteristics of 99 cases of 2019 novel coronavirus pneumonia in Wuhan, China: a descriptive study. Lancet (London, England) 2020; 395:507- 
13.

11. Liu H, J Li, M Chen, J Su. Glucocorticoid treatment of suspected organizing pneumonia after H7N9 infection: A case report. Medicine 2019; 98:e16839.

12. Organization World Health. Clinical management of severe acute respiratory infection when novel coronavirus (nCoV) infection is suspected: interim guidance, 25 January 2020. Geneva: World Health Organization, 2020.

13. Kidney disease: improving global outcomes (KDIGO) acute kidney injury work group. KDIGO clinical practice guideline for acute kidney injury. March 2012. https://kdigo.org/wp-content/ uploads/2016/10/KDIGO-2012-AKI-Guideline-English.pdf (accessed Feb 8, 2020).

14. National Health Commission of the People's Republic of China. Guidelines for the Diagnosis and Treatment of Novel Coronavirus (2019-nCoV) Infection by the National Health Commission (Trial Version 5) 2020. (Accessed Jan 282020 at http://www.chinacdc.cn/jkzt/crb/zl/szkb_11803/jszl_11815/202001/W020200128207842237479.pdf).

15. Hansell DM, AA Bankier, H MacMahon, TC McLoud, NL Müller, J Remy. Fleischner Society: glossary of terms for thoracic imaging. Radiology 2008; 246:697-722.

16. Schoen K, N Horvat, NFC Guerreiro, I de Castro, KS de Giassi. Spectrum of clinical and radiographic findings in patients with diagnosis of $\mathrm{H} 1 \mathrm{~N} 1$ and correlation with clinical severity. BMC infectious diseases 2019; 19:964.

17. Shang L, J Zhao, Y Hu, R Du, B Cao. On the use of corticosteroids for 2019-nCoV pneumonia. Lancet (London, England) 2020; 395:683-4.

18. Cruz-Topete D, JA Cidlowski. One hormone, two actions: anti- and pro-inflammatory effects of glucocorticoids. Neuroimmunomodulation 2015; 22:20-32.

19. Vandewalle J, A Luypaert, K De Bosscher, C Libert. Therapeutic Mechanisms of Glucocorticoids. Trends in endocrinology and metabolism: TEM 2018; 29:42-54.

20. Xu Z, L Shi, Y Wang, et al. Pathological findings of COVID-19 associated with acute respiratory distress syndrome. The Lancet Respiratory medicine 2020; 8:420-2.

21. Jing Liu, Li Sumeng, Liu Jia, et al. Longitudinal characteristics of lymphocyte responses and cytokine profiles in the peripheral blood of SARS-CoV-2 infected patients. medRxiv 2020:2020.02.16.20023671.

22. Bo Diao, Wang Chenhui, Tan Yingjun, et al. Reduction and Functional Exhaustion of T Cells in Patients with Coronavirus Disease 2019 (COVID-19). medRxiv 2020:2020.02.18.20024364.

23. Ghoneim HE, JA McCullers. Adjunctive corticosteroid therapy improves lung immunopathology and survival during severe secondary pneumococcal pneumonia in mice. The Journal of infectious diseases 2014; 209:1459-68.

24. Schäcke H, WD Döcke, K Asadullah. Mechanisms involved in the side effects of glucocorticoids. Pharmacology \& therapeutics 2002; 96:23-43.

25. Rhen T, JA Cidlowski. Antiinflammatory action of glucocorticoids-new mechanisms for old drugs. The New England journal of medicine 2005; 353:1711-23. 
26. Wilkinson TM, CK Li, CS Chui, et al. Preexisting influenza-specific CD4+ T cells correlate with disease protection against influenza challenge in humans. Nature medicine 2012; 18:274-80.

27. Li YM, SX Wang, HS Gao, et al. [Factors of avascular necrosis of femoral head and osteoporosis in SARS patients' convalescence]. Zhonghua yi xue za zhi 2004; 84:1348-53.

28. Brun-Buisson C, JC Richard, A Mercat, AC Thiébaut, L Brochard. Early corticosteroids in severe influenza A/H1N1 pneumonia and acute respiratory distress syndrome. American journal of respiratory and critical care medicine 2011; 183:1200-6.

29. Stockman LJ, R Bellamy, P Garner. SARS: systematic review of treatment effects. PLoS medicine 2006; 3:e343.

30. Lee N, KC Allen Chan, DS Hui, et al. Effects of early corticosteroid treatment on plasma SARS-associated Coronavirus RNA concentrations in adult patients. Journal of clinical virology : the official publication of the Pan American Society for Clinical Virology 2004; 31:304-9.

31. Arabi YM, Y Mandourah, F Al-Hameed, et al. Corticosteroid Therapy for Critically III Patients with Middle East Respiratory Syndrome. American journal of respiratory and critical care medicine 2018; 197:757-67.

32. Ni YN, G Chen, J Sun, BM Liang, ZA Liang. The effect of corticosteroids on mortality of patients with influenza pneumonia: a systematic review and meta-analysis. Critical care (London, England) 2019; 23:99.

33. Rhodes A, LE Evans, W Alhazzani, et al. Surviving Sepsis Campaign: International Guidelines for Management of Sepsis and Septic Shock: 2016. Critical care medicine 2017; 45:486-552.

34. Marik PE. Steroids for sepsis: yes, no or maybe. J Thorac Dis 2018; 10:S1070-S3.

35. Wang D, B Hu, C Hu, et al. Clinical Characteristics of 138 Hospitalized Patients With 2019 Novel Coronavirus-Infected Pneumonia in Wuhan, China. Jama 2020.

36. Zhou W, Y Liu, D Tian, et al. Potential benefits of precise corticosteroids therapy for severe 2019-nCoV pneumonia. Signal transduction and targeted therapy 2020; 5:18.

37. Xie LX, YN Liu, FY Hao, et al. [Prognostic analysis of lung function and chest X-ray changes of 258 patients with severe acute respiratory syndrome in rehabilitation after discharge]. Zhonghua jie he he hu xi za zhi = Zhonghua jiehe he huxi zazhi = Chinese journal of tuberculosis and respiratory diseases 2004; 27:147-50.

38. Meng QH, PL Dong, YB Guo, et al. [Use of glucocorticoid in treatment of severe acute respiratory syndrome cases]. Zhonghua yu fang yi xue za zhi [Chinese journal of preventive medicine] 2003; 37:233-5.

39. Zhao ZW, FC Zhang, M Xu, et al. [Clinical analysis of 190 cases of outbreak with atypical pneumonia in Guangzhou in spring, 2003]. Zhonghua yi xue za zhi 2003; 83:713-8.

\section{Tables}

Table 1. Demographics and baseline characteristics of patients with severe COVID-19. 


\section{Variables}

Total $(n=75) \quad \begin{aligned} & \text { Corticosteroid group } \\ & (n=47)\end{aligned}$

Non-Corticosteroid group

$(n=28)$

value

\section{Demographic factors}

\begin{tabular}{|c|c|c|c|c|}
\hline Male (\%) & $39(52.00)$ & 25 (53.19) & $14(50.00)$ & 0.789 \\
\hline Age (\%) & $58.92 \pm 14.29$ & $57.09 \pm 13.74$ & $62.00 \pm 14.90$ & 0.151 \\
\hline Smoking (\%) & 9 (12.00) & $8(17.02)$ & $1(3.57)$ & 0.083 \\
\hline APACHE II & $7.97 \pm 3.37$ & $8.24 \pm 3.57$ & $7.52 \pm 3.02$ & 0.382 \\
\hline \multicolumn{5}{|l|}{ Comorbidity } \\
\hline Hypertension (\%) & 13 (17.33) & $7(14.89)$ & $6(21.43)$ & 0.470 \\
\hline Diabetes (\%) & 19 (25.33) & $13(27.66)$ & $6(21.43)$ & 0.548 \\
\hline CHD (\%) & $4(5.33)$ & $2(4.26)$ & $2(7.14)$ & 0.590 \\
\hline COPD (\%) & 7 (9.33) & $3(6.38)$ & $4(14.28)$ & 0.437 \\
\hline $\begin{array}{l}\text { Cerebrovascular } \\
\text { disease (\%) }\end{array}$ & $1(1.33)$ & $1(2.13)$ & 0 & 1.000 \\
\hline Viral hepatitis (\%) & $1(1.33)$ & $1(2.13)$ & 0 & 1.000 \\
\hline Tumor (\%) & $2(2.67)$ & $1(2.13)$ & $1(3.57)$ & 0.707 \\
\hline CKD (\%) & $1(1.33)$ & $1(2.13)$ & 0 & 1.000 \\
\hline \multicolumn{5}{|l|}{ Symptoms } \\
\hline Fever (\%) & 46 (61.33) & 31 (65.96) & $15(53.57)$ & 0.287 \\
\hline Cough (\%) & $57(76.00)$ & 37 (78.72) & 20 (71.43) & 0.474 \\
\hline Sputum (\%) & 26 (34.67) & 15 (31.91) & 11 (39.29) & 0.516 \\
\hline dyspnea (\%)\ & 40 (53.33) & 30 (63.83) & $10(35.71)$ & 0.018 \\
\hline Weakness (\%) & $22(29.33)$ & $12(25.53)$ & $10(35.71)$ & 0.349 \\
\hline Headache (\%) & 8 (10.67) & $4(8.51)$ & $4(14.28)$ & 0.433 \\
\hline Diarrhea (\%) & $6(8.00)$ & $3(6.38)$ & $3(10.71)$ & 0.819 \\
\hline \multicolumn{5}{|l|}{ Complication } \\
\hline Shock (\%) & $3(4.00)$ & $3(6.38)$ & 0 & 0.450 \\
\hline Leukopenia (\%) & $4(5.33)$ & $3(6.38)$ & $1(3.57)$ & 0.600 \\
\hline Thrombocytopenia (\%) & $2(2.67)$ & $2(4.26)$ & 0 & 0.715 \\
\hline Liver dysfunction (\%) & $3(4.00)$ & $2(4.26)$ & $1(3.57)$ & 0.884 \\
\hline AKI (\%) & $2(2.67)$ & $1(2.13)$ & 1 (3.57) & 0.707 \\
\hline
\end{tabular}


Note: $\mathrm{AKI}=$ Acute kidney injury; $\mathrm{APACHE}=$ acute physiology and chronic health assessment, $\mathrm{CHD}=$ coronary heart disease, $\mathrm{COPD}=$ chronic obstructive pulmonary disease, $\mathrm{CKD}=$ chronic kidney disease, SOFA = sequential organ failure assessment.

Table 2. Treatments and outcomes in patients with severe COVID-19.

\begin{tabular}{|c|c|c|c|c|}
\hline Variables & $\begin{array}{l}\text { Total } \\
(n=75)\end{array}$ & $\begin{array}{l}\text { Corticosteroid } \\
\text { group } \\
(n=47)\end{array}$ & $\begin{array}{l}\text { Non-corticosteroid group } \\
(n=28)\end{array}$ & $p$-value \\
\hline \multicolumn{5}{|l|}{ Treatments } \\
\hline Antibiotics (\%) & $62(82.67)$ & 45 (95.74) & $17(60.71)$ & $<0.001$ \\
\hline Antifungal (\%) & $12(16.00)$ & $12(25.53)$ & 0 & 0.004 \\
\hline IV immunoglobulin (\%) & $30(42.76)$ & 27 (57.45) & $3(10.71)$ & $<0.001$ \\
\hline $\begin{array}{l}\text { Thymopentin or } \\
\text { thymalfasin (\%) }\end{array}$ & $63(84.00)$ & 43 (91.49) & $20(71.43)$ & 0.022 \\
\hline High-flow oxygen (\%) & $40(53.33)$ & $28(59.57)$ & $12(42.86)$ & 0.160 \\
\hline Noninvasive ventilator (\%) & 28 (37.33) & $24(51.06)$ & $4(14.28)$ & 0.001 \\
\hline Invasive ventilator (\%) & $7(9.33)$ & $7(14.89)$ & 0 & 0.032 \\
\hline ECMO (\%) & $3(4.00)$ & $3(6.38)$ & 0 & 0.450 \\
\hline \multicolumn{5}{|l|}{ Outcomes } \\
\hline Total length of hospital stay & $21.05 \pm 8.96$ & $21.21 \pm 9.57$ & $20.79 \pm 7.98$ & 0.843 \\
\hline Length of ICU stay & $14.64 \pm 6.67$ & $15.23 \pm 6.72$ & $13.64 \pm 6.58$ & 0.321 \\
\hline High-flow oxygen days & $5.58 \pm 4.19$ & $5.32 \pm 3.58$ & $6.17 \pm 5.49$ & 0.565 \\
\hline Noninvasive ventilator days & $6.69 \pm 5.37$ & $7.54 \pm 5.46$ & $2.60 \pm 2.30$ & 0.060 \\
\hline Invasive ventilator days & $9.00 \pm 6.14$ & $9.00 \pm 6.14$ & - & - \\
\hline Mortality rate (\%) & $4(5.33)$ & $4(8.51)$ & 0 & 0.291 \\
\hline
\end{tabular}

Note: IV = intravenous injection, ECMO = extracorporeal membrane oxygenation, ICU= intensive care unit. 
Table 3. Comparison of laboratory variables at different follow-up points in patients with severe COVID-19.

\begin{tabular}{|c|c|c|c|c|}
\hline \multirow[t]{2}{*}{ Variables } & \multicolumn{2}{|c|}{ Corticosteroid group } & \multicolumn{2}{|c|}{ Non-corticosteroid group } \\
\hline & D 0 & D 14 & D 0 & D 14 \\
\hline Heart rate (bpm) & $84 \pm 16$ & $80 \pm 16$ & $88 \pm 15$ & $74 \pm 6 \#$ \\
\hline Respiratory rate & $24 \pm 12$ & $20 \pm 2$ & $20 \pm 2$ & $20 \pm 2$ \\
\hline MAP $(\mathrm{mmHg})$ & $90 \pm 10$ & $87 \pm 11$ & $91 \pm 12$ & $82 \pm 21 \#$ \\
\hline $\begin{array}{l}\text { Body temperature } \\
\left({ }^{\circ} \mathrm{C}\right)\end{array}$ & $37.4 \pm 1.0$ & $36.8 \pm 0.5 \#$ & $37.3 \pm 0.8$ & $36.5 \pm 0.3 \#$ \\
\hline APACHE II & $8.24 \pm 3.57$ & $6.72 \pm 4.87 \#$ & $7.52 \pm 3.02$ & $6.08 \pm 3.39 \#$ \\
\hline $\begin{array}{l}\text { P/F Ratio } \\
(\mathrm{mmHg})\end{array}$ & $196.68 \pm 105.70$ & $297.47 \pm 135.68 \#$ & $239.00 \pm 107.76$ & $308.20 \pm 102.54 \#$ \\
\hline WBC $\left(\times 10^{9} / \mathrm{L}\right)$ & $6.06 \pm 3.17$ & $6.87 \pm 2.70$ & $6.24 \pm 2.72$ & $6.36 \pm 1.68$ \\
\hline$N\left(\times 10^{9} / L\right)$ & $4.91 \pm 3.11$ & $5.46 \pm 2.67$ & $4.75 \pm 2.57$ & $4.11 \pm 1.11$ \\
\hline$L\left(\times 10^{9} / L\right)$ & $0.79 \pm 0.34^{*}$ & $0.84 \pm 0.54^{*}$ & $1.02 \pm 0.39$ & $1.13 \pm 0.31$ \\
\hline РCT (ng/mL) & $0.14 \pm 0.13$ & $0.12 \pm 0.11$ & $0.07 \pm 0.05$ & $0.05 \pm 0.04 \#$ \\
\hline ALT (U/L) & $53.86 \pm 45.69$ & $56.18 \pm 52.36$ & $27.06 \pm 14.50$ & $38.42 \pm 26.58 \#$ \\
\hline AST (U/L) & $52.39 \pm 44.33$ & $60.15 \pm 49.77$ & $31.78 \pm 10.39$ & $29.49 \pm 17.07$ \\
\hline LDH (U/L) & $\begin{array}{l}365.63 \pm \\
169.73\end{array}$ & - & $321.50 \pm 85.82$ & - \\
\hline Albumin $(\mathrm{g} / \mathrm{L})$ & $36.27 \pm 4.25$ & $34.12 \pm 3.28$ & $37.49 \pm 3.93$ & $33.21 \pm 3.64 \#$ \\
\hline TB $(\mu \mathrm{mol} / \mathrm{L})$ & $18.22 \pm 6.59$ & - & $14.43 \pm 6.96$ & - \\
\hline CRP (mg/L) & $83.19 \pm 63.09$ & $27.15 \pm 20.05 \#$ & $70.92 \pm 61.73$ & $18.64 \pm 11.09 \#$ \\
\hline D-dimer (mg/L) & $1.86 \pm 1.42$ & $4.03 \pm 5.64 \#$ & $0.58 \pm 0.47$ & $1.01 \pm 0.89$ \\
\hline IL $10(p g / m L)$ & $4.51 \pm 2.37$ & $6.99 \pm 6.35 \#$ & $4.68 \pm 1.37$ & $3.47 \pm 0.78 \#$ \\
\hline IL 6 (pg/mL) & $43.35 \pm 34.19$ & $40.16 \pm 28.61$ & $33.03 \pm 23.69$ & $31.86 \pm 27.13$ \\
\hline $\begin{array}{l}\text { CD 4+ T (cells/ } \\
\mu \mathrm{L})\end{array}$ & $253.20 \pm 144.84$ & $374.76 \pm 195.80 \#$ & $298.31 \pm 164.52$ & $430.20 \pm 161.40 \#$ \\
\hline $\begin{array}{l}\text { CD 8+ T (cells/ } \\
\mu \mathrm{L})\end{array}$ & $157.55 \pm 103.76^{*}$ & $285.95 \pm 248.60 \#$ & $220.55 \pm 110.87$ & $275.29 \pm 108.06 \#$ \\
\hline CD 4/CD 8 Ratio & $1.70 \pm 0.84$ & $1.91 \pm 1.39 \#$ & $1.51 \pm 0.63$ & $1.68 \pm 0.61$ \\
\hline PT (s) & $11.70 \pm 1.30$ & $11.41 \pm 1.76$ & $12.03 \pm 2.16$ & $11.42 \pm 0.74$ \\
\hline $\mathrm{Cr}(\mu \mathrm{mol} / \mathrm{L})$ & $64.51 \pm 17.13$ & $66.99 \pm 24.80$ & $68.94 \pm 30.99$ & $73.91 \pm 50.68$ \\
\hline
\end{tabular}


Note: $\mathrm{ALT}=$ alanine aminotransferase, $\mathrm{APACHE}=$ acute physiology and chronic health assessment, $\mathrm{AST}=$ aspartate aminotransferase, $\mathrm{CD}=$ clusters of differentiation, $\mathrm{Cr}=$ creatinine, $\mathrm{CRP}=\mathrm{C}$-reactive protein, IL = interleukin, $\mathrm{L}=\mathrm{Lymphocyte}$ count, $\mathrm{LDH}=$ lactic dehydrogenase, $\mathrm{MAP}=$ mean arterial pressure $\mathrm{N}=$ neutrophil count, $\mathrm{P} / \mathrm{F}$ Ratio $=\mathrm{PaO}_{2} / \mathrm{FiO}_{2}$ or oxygenation index, $\mathrm{PCT}=$ procalcitonin, $\mathrm{PT}=$ prothrombin time, $\mathrm{s}=$ second, SOFA $=$ sequential organ failure assessment, $T=T$ cell, $T B=$ total bilirubin, $W B C=$ white blood cell. ${ }^{*} p<0.05$ compare with Non-corticosteroid group. \# $p<0.05$ compare with baseline (D 0$)$ in each group.

Table 4. The results of imaging evaluation in patients with COVID-19.

\begin{tabular}{|lllll|}
\hline Variables & \multicolumn{2}{l}{ Corticosteroid group } & \multicolumn{2}{l|}{ Non-corticosteroid group } \\
\cline { 2 - 5 } & D 0 & D 14 & D 0 & D 14 \\
\hline Total lesion volume ratio (\%) & $22.14 \pm 12.62^{\star}$ & $11.21 \pm 8.17 \#$ & $12.80 \pm 8.32$ & $12.05 \pm 9.44$ \\
\hline Consolidation volume ratio (\%) & $4.59 \pm 4.46^{*}$ & $2.77 \pm 3.99 \#$ & $1.68 \pm 1.47$ & $2.81 \pm 2.87 \#$ \\
\hline GGO volume ratio (\%) & $17.26 \pm 12.35^{\star}$ & $8.31 \pm 5.73 \#$ & $11.30 \pm 7.85$ & $9.15 \pm 7.63$ \\
\hline
\end{tabular}

Note: $\mathrm{D}=$ day, $\mathrm{GGO}=$ ground-glass opacity, $\mathrm{w}=$ week. ${ }^{*} p<0.05$ compare with Non-corticosteroid group. $\# p<$ 0.05 compare with baseline (D 0$)$ in each group.

\section{Figures}



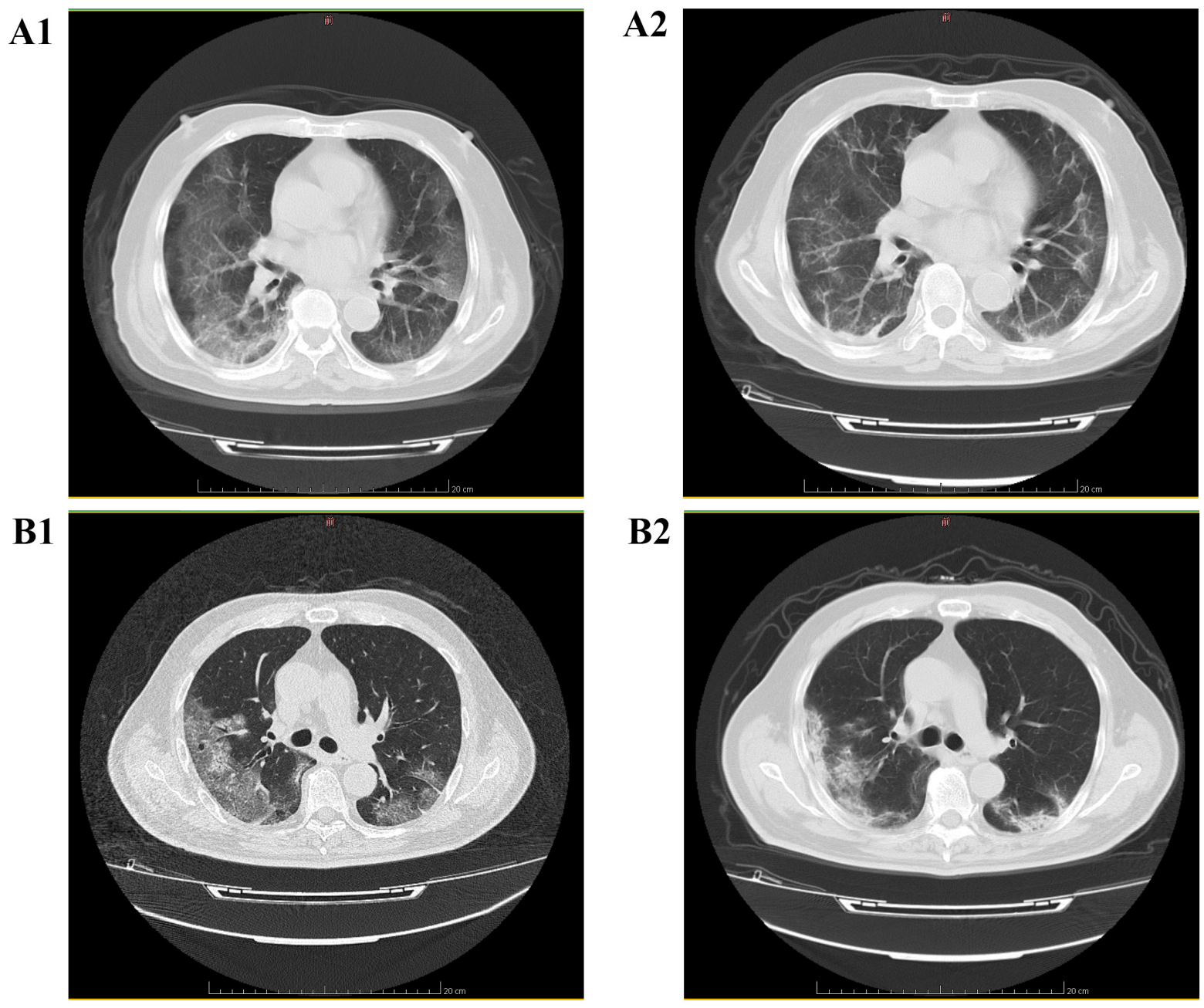

B2

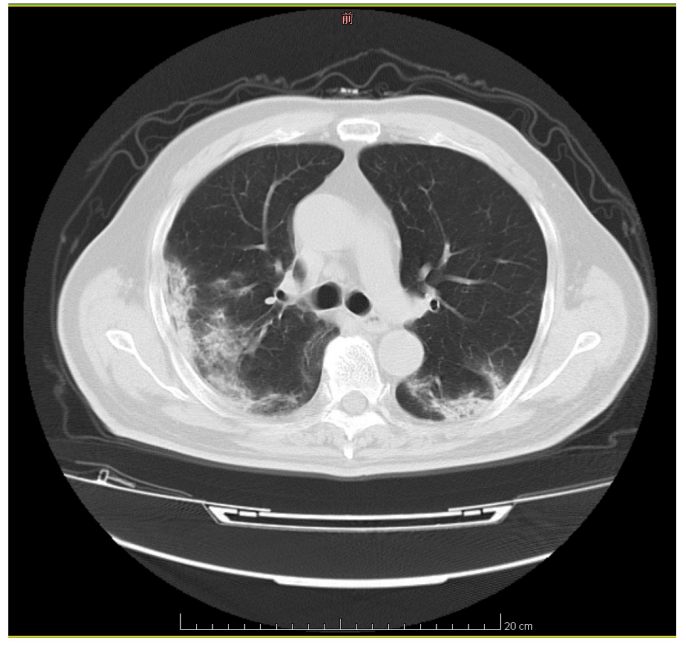

D0

D14

\section{Corticosteroid group: A1 and A2; Non-corticosteroid group: B1 and B2}

\section{Figure 1}

CT manifestations of lung tissue in the patients with severe COVID 19. A1 and A2 were corticosteroid group, B1 and B2 were non-corticosteroid group. 